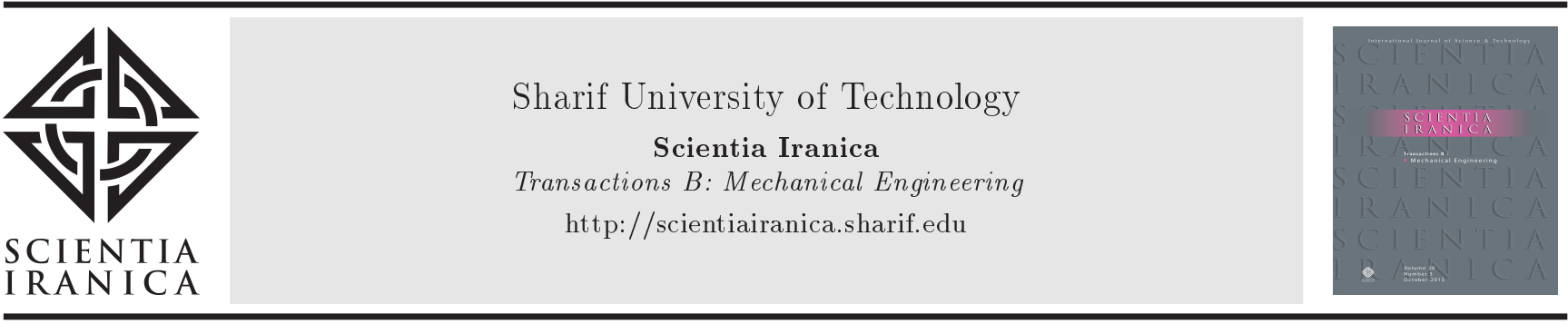

\title{
Numerical simulation of a neuron under blast load using viscoelastic material models
}

\author{
H. Ahmadi Nejad Joushani ${ }^{a}$, B. Vahidi ${ }^{b, *}$, and M.-H. Sabour ${ }^{a, *}$ \\ a. Department of Aerospace Engineering, Faculty of New Sciences and Technologies, University of Tehran, Tehran, Iran. \\ b. Division of Biomedical Engineering, Department of Life Science Engineering, Faculty of New Sciences and Technologies, \\ University of Tehran, Tehran, Iran.
}

Received 24 October 2018; received in revised form 5 October 2019; accepted 28 December 2019

\section{KEYWORDS}

Blast load;

Cell mechanics;

Finite elements;

Neuronal cell;

Traumatic brain

injury.

\begin{abstract}
Traumatic Brain Injury (TBI) is usually caused by physical brain injury. The present study proposes a computational model that considers the response of a neuronal cell under blast loading. The neuronal cell consists of four components including nucleus, cytoplasm, membrane, and a network of microtubules with different arrays such as crossing, stellate, and random orientations. This study investigates the effect of sub-cellular components, specifically the network of microtubules, on the consequences of a TBI, which can be regarded as a novel innovation. While nucleus, cytoplasm, and membrane were assumed viscoelastic, the network of microtubules exhibited elastic behavior. Finite element methods and fluid-structure interactions were employed to solve the coupled equations of both solid and fluid. The results showed that the presence of a network of microtubules, regardless of the types of arrays, could reduce the total displacement of the cell and von Mises stress, as well. In this study, the membrane von Mises stress decreased by $50 \%$ from 30 to 15 Pascal in the presence of the network of the microtubules. The obtained results could be used in different fields including treatment of some diseases and pathological conditions such as kidney stones, sports injuries, traumatic astronauts, and ultimately prevention and treatment of traumatic brain injuries.
\end{abstract}

(C) 2021 Sharif University of Technology. All rights reserved.

\section{Introduction}

Different mechanical loads on the brain such as head injury, vehicle accident, sports injuries, and blast waves cause Traumatic Brain Injury (TBI). All of these forces could provoke a mechanical response from the brain. Such a response would stimulate pathophysiological responses from the brain and consequently, lead to brain damages as well as behavioral and cognitive changes.

\footnotetext{
*. Corresponding authors. Tel.: 00982186093021 ; Fax: 00982188617087

E-mail addresses: bahman.vahidi@ut.ac.ir (B. Vahidi); sabourmh@ut.ac.ir (M.H.Sabour)
}

doi: $10.24200 /$ sci. 2019.51975 .2456
Damages to human beings on all scales from lungs, eyes, ears, heart, brain to organs, tissues, cells, and sub-cellular components have been reported as blast injuries up until now [1]. Therefore, blast waves affect the human body at physiological, cellular, sub-cellular, and molecular levels. Numerous researchers have made many attempts to understand the mechanism for brain damages at the cellular level [2]. TBI has been recently considered as one of the most significant factors related to brain misfunctioning. In addition to the need for further laboratory research, an urgent requirement for developing a computational model is also felt in order to better understand the cell behavior while responding to such loading [3].

A 3D computational framework was presented by Jérusalem and Dao [2] to evaluate the neuronal cell 
response under blast load. The maximum von Mises stress for the cell parts was reported as $19.8,5.5$, and $19.4 \mathrm{~Pa}$ for the membrane, cytoplasm, and nucleus, respectively. Both computational and experimental approaches to single cortical neurons were presented by Bernick et al. [4]. The geometric parameters of the cell were derived from the atomic force microscopy results and a three-dimensional Finite Element (FE) model of a single neuron cell was extracted. Eslaminejad et al. [5] studied the tissue dynamic response under shock waves using three different material models including hyperviscoelastic, hyperelastic, and viscoelastic. They captured the maximum intracranial pressure, maximum principal strain, and maximum principal stress. It was indicated that the results for intracranial pressure and principal strain were pretty similar to each other for three constitutive models, especially for viscoelastic and hyperviscoelastic material behaviors. The principal shear stress was predicted differently for three material models. Shams et al. [6] performed a FE analysis to predict the dynamic response of foam-filled aluminum parabolic tubes to axial impact loading. The effect of geometric and material parameters on the response of the tubes under impact was investigated. They concluded that the foam properties had a significant effect on the dynamic response of the tubes. Ganpule et al. [7] investigated the effect of the blast wave on the dummy and real human head models using numerical and experimental methods. It was proved that the surface pressures and velocities were affected by head geometries and these surface pressures played a key role in the deformations and stresses inside the brain. Laksari et al. [8] studied the nonlinear wave propagation and shock formation in the brain tissue using numerical methods. It was observed that the stress wave propagation could result in a shock wave within the brain tissue. This could bring about large stress and strain within the brain tissue which would cause high gradient-induced injury. In another research performed by Laksari et al. [9], the underlying physics of wave propagation in the brain tissue was analyzed. The results revealed that tissue nonlinearity and rate dependence were of importance in such mechanical loading. In addition, the high spatial gradients and high rates of strain and stress at the shock front were responsible for neuronal cell injury.

Taylor et al. [10] developed a human head model to determine the effect of stress wave energy resulting from blast load on the brain. It was predicted that shear stress and cavitation resulting from the stress wave, independent of the wave direction, could be considered as major injury mechanisms. Teferra et al. [11] proposed a methodology to evaluate the effect of morphological variability on the mechanical response of the head under blast loading. As a result, it was demonstrated that the magnitude of peak overpressure was dependent on the head morphological variability; however, the location of the overpressure did not change with morphological variability. Additionally, it was deduced that the strain was more sensitive to morphological variability than pressure. Ganpule et al. [12] examined the effect of bulk modulus on a 3D computational human head model. It was reported that a bulk modulus in the order of GPa yielded more accurate results based on experimental data. Tan et al. [13] suggested a detailed human body FE model to determine the biomechanical response and the mechanism of brain injury during blast loading. It was demonstrated that the human head model could not estimate the exact strain rate inside the brain. Rodríguez-Millán et al. [14] recommended a 3D head helmet numerical model to investigate the head response under blast load. The results of their research indicated that a single protective helmet was not sufficient to reduce brain damage and instead, a complete protective helmet would be more useful to prevent skull fracture and high pressure in the brain. Finan [15] investigated the biomechanical simulation of TBI in the rat so as to understand TBI injury mechanism. It was reported that data associated with the biomechanical properties of the rat brain increased dramatically over the past few years; therefore, new biomechanical models have emerged quite differently from the traditional ones and created new biomechanical challenges. It has been concluded that there is an urgent need for predicting the accurate set of data that could be used for validation of the FE models of the brain interior motion. Palombo et al. [16] proposed a controlled, flexible, novel generative model of the brain cell to simulate molecular diffusion within the neurons. It was deduced that their computational framework successfully addressed the main challenges through numerical simulation of the diffusion process within the brain cell. Furthermore, the versatility and potentiality of the computational framework were demonstrated by selecting a set of examples. They presented the first step in investigating the morphology of the brain cell. In the research carried out by LU et al. [17], a 3D computational head model under dynamic head rotation as well as extension was employed. A subjectspecific computational head model with anisotropic properties of the white matter was built from T1 magnetic resonance images, which could predict strains in the brain under large rotational acceleration. It was reported that their computational model was able to predict the observed strain fields better than a model without falx and tentorium for the axial and sagittal rotations under mild angular acceleration.

In order to study the mechanical response of the neuronal cell, at first, it is essential to have a proper model of a neuronal cell to guarantee the accuracy of the results. The complex structure of 
the neuronal cell and the complexity of its internal components have made precise simulation extraordinarily difficult. The computational techniques require accurate knowledge of the cell geometry and material parameters. Therefore, uncovering the molecular and cellular mechanisms of TBI would require a careful investigation. This research as indicated in Ahmadi Nejad Joushani et al. [18] aims to predict the effects of mechanical load on the neuronal cell on the subcellular scale, especially on the network of microtubules within the cytoplasm of the neuronal cell as one of the major sub-cellular components. To the best of our knowledge, simulation of the network of microtubules as sub-cellular components in TBI research still has not been performed so far. In addition to the three main parts of the cell including nucleus, membrane, and cytoplasm, a network of microtubules with different arrays including crossing and stellate as well as random orientations was considered. The load was chosen such that it would resemble the reality of the shock wave [19].

\section{Material and methods}

\subsection{Geometry and boundary conditions}

An investigation was carried out on the neuronal cell geometry and material parameters. The parameters were extracted from the literature [2,4]. The overall cell geometry consisted of three main parts and three different arrays of the microtubule network. The height and the diameter of the cell were chosen as 7.9 and 16.8 micrometers, respectively [4]. The thickness of the membrane was considered 200 nanometers [20,21]. The nucleus of the cell was characterized by a diameter of 11.65 micrometers and a height of 5.48 micrometers [4]. Figure 1a. shows the overall cell geometry with the above-mentioned dimensions. The network of microtubules in this study was in accordance with the dimensions mentioned in the work done by O'Connor et

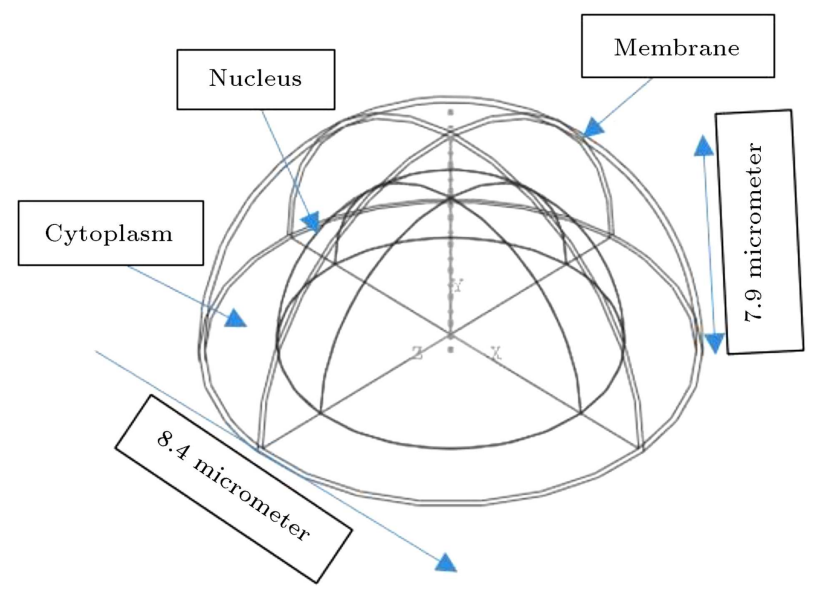

Figure 1a. Overall cell geometry schematic.

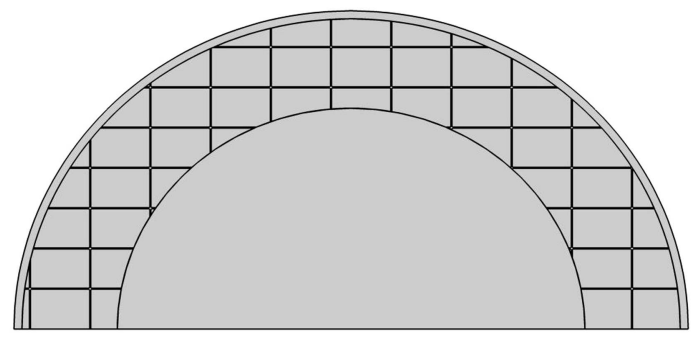

Figure 1b. Cell schematic with a crossing array of a network of microtubules.

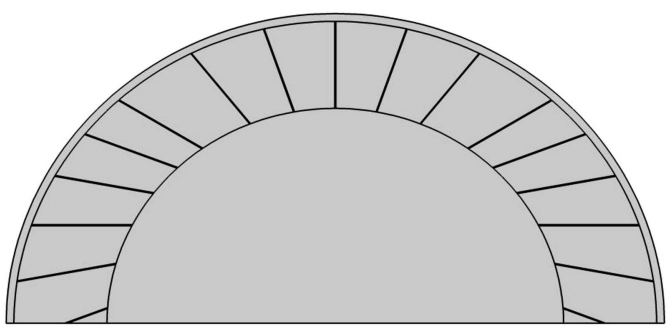

Figure 1b. Cell schematic with a stellate array of a network of microtubules.

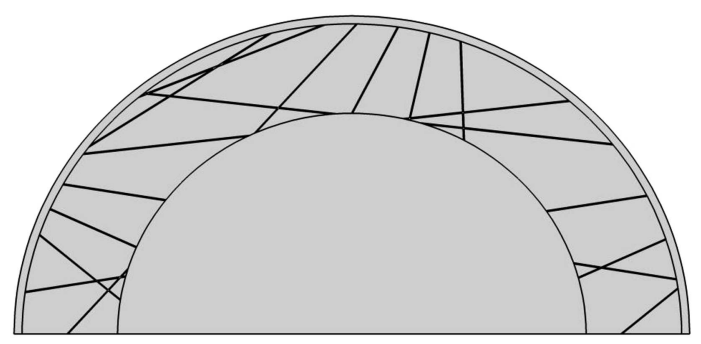

Figure 1d. Cell schematic with a random orientation array of a network of microtubules.

al. [22], had a diameter of 25 nanometers and different lengths, and the filaments were connected in three different arrangements. Figures $1 \mathrm{~b}$ to $1 \mathrm{~d}$ show an overview of the model of the cell with various arrays of the microtubule networks inside the cytoplasm. Figures $1 \mathrm{~b}$ to $1 \mathrm{~d}$ show the cells with crossing, stellate, and random orientation arrays of the microtubule networks, respectively.

In laboratory studies in which a laser-induced pressure system is used to study the cell response, the cell is immersed in fluid [23]. Therefore, to compare the simulation with laboratory experiments [19], the boundary and loading conditions were considered in such a way they could be similar to real experimental conditions. For this purpose, a cube of $100 \times 100 \mu \mathrm{m}^{2}$ of water was considered and the cell was placed on the cubic floor (see Figure 2). The upper wall of the cube was exposed to blast load, while symmetry boundary conditions were assumed for the four remaining walls. The bottom of the cell was fixed to the cube wall and the FSI (Fluid-Structure Interaction) boundary condition was considered between the cell surface and the surrounding fluid. Furthermore, the 


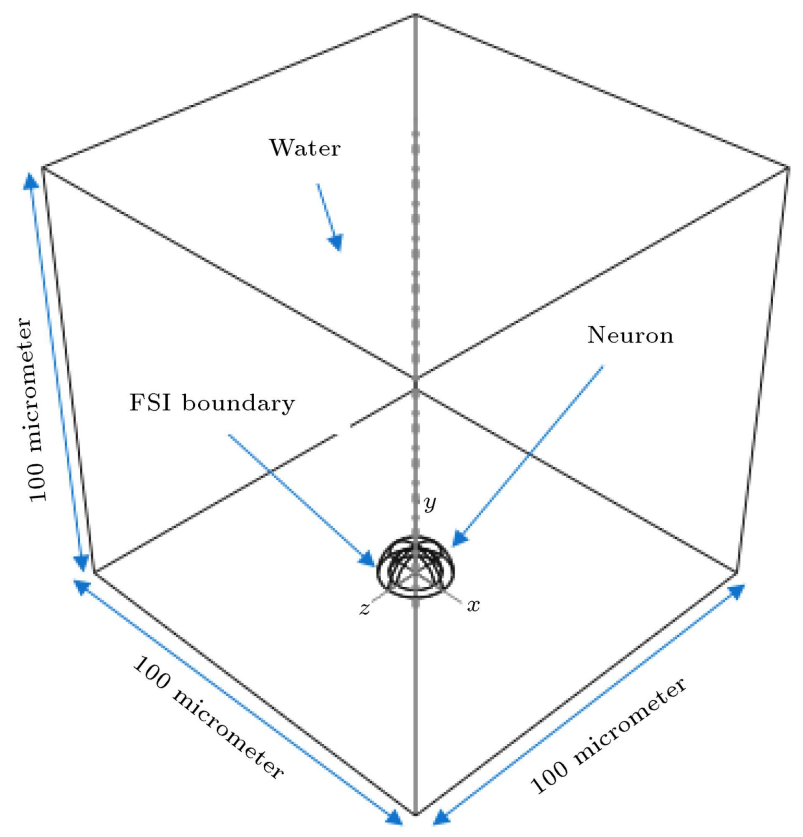

Figure 2. Overall geometry of the cell with cube.

kinematic equilibrium boundary conditions (the equality of displacements at the boundary) and the dynamic equilibrium boundary conditions (equality of stresses at the boundary) were applied to the FSI boundary. The cell and the cube were symmetrical with respect to the vertical axis.

To compare the results with findings of the former study [2], a blast load was considered with maximum pressure of $50 \mathrm{MPa}$ and a duration of 8 nanoseconds, which had the positive phase of one nanosecond (Figure 3) [19].

Figures 4a to $4 \mathrm{~d}$ show the FE discretization of the overall model, cell with crossing array, cell with stellate array, and cell with random orientation arrays.

\subsection{Material parameters}

The nucleus, cytoplasm, and membrane exhibited viscoelastic material behavior. In this regard, the firstorder generalized Maxwell model was implemented for

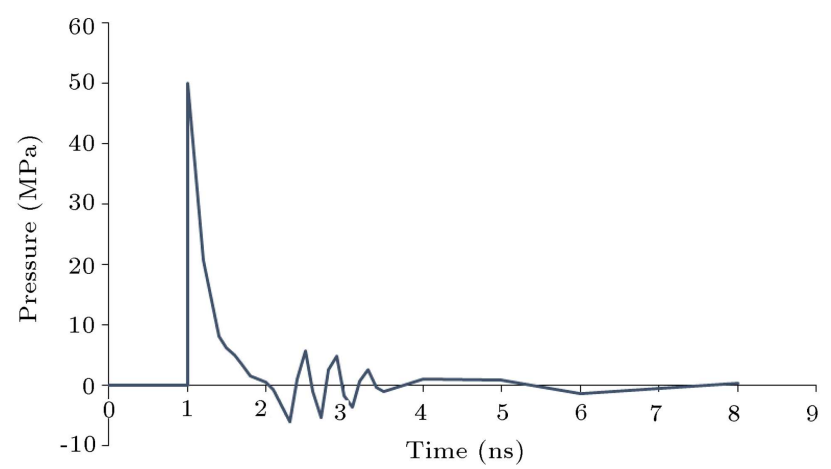

Figure 3. Schematic configuration of blast load changes with time [19].

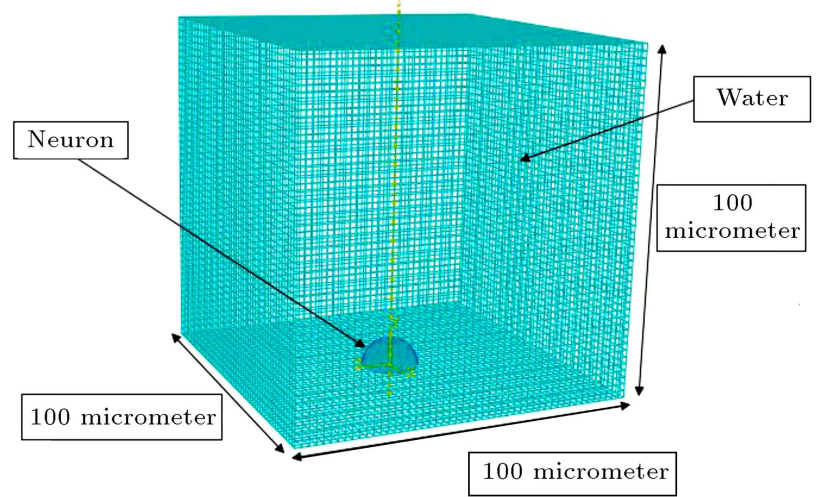

Figure 4a. Finite element discretization of the overall model.

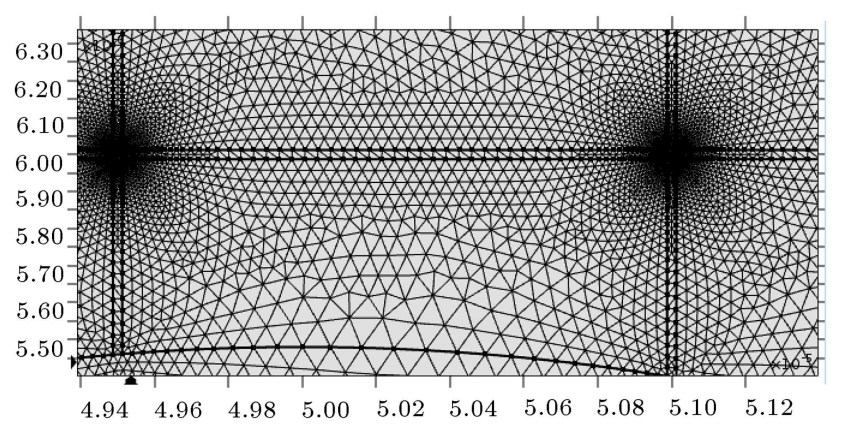

Figure $4 \mathrm{~b}$. Finite element discretization of the cell with a crossing array of a network of microtubules.

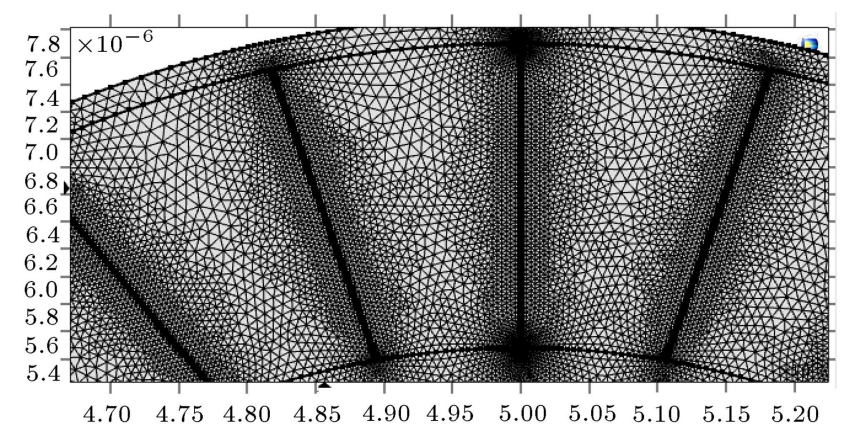

Figure 4c. Finite element discretization of the cell with stellate array of a network of microtubules.

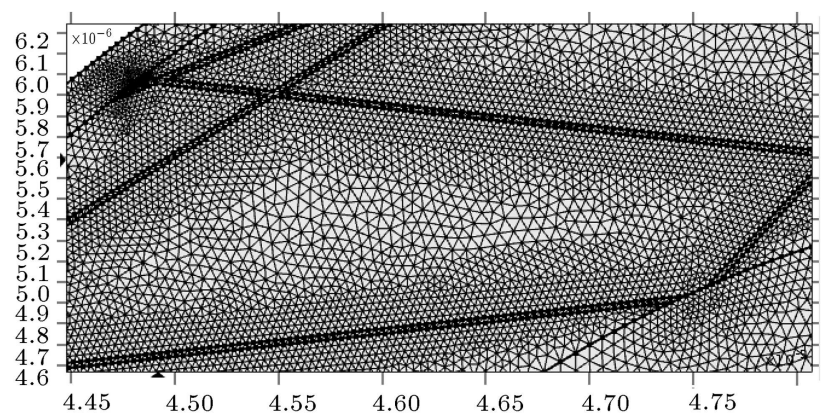

Figure 4d. Finite element discretization of the cell with a random orientation array of a network of microtubules. 
Table 1. Viscoelastic parameters for the cell parts.

\begin{tabular}{lccc}
\hline Cell parts & $\begin{array}{c}\text { Shear } \\
\text { modulus } \\
(\mathbf{P a})\end{array}$ & $\begin{array}{c}\text { Relaxation } \\
\text { time } \\
(\mathbf{s})\end{array}$ & $\begin{array}{c}\text { Viscosity } \\
\text { (Pa.s) }\end{array}$ \\
\hline Membrane & 13.89 & 3000 & 41.67 \\
Cytoplasm & 107.78 & 0.01 & 1.08 \\
Nucleus & 862.22 & 0.5 & 431.11 \\
[Ref.] & {$[5]$} & {$[5]$} & {$[5]$} \\
\hline
\end{tabular}

nucleus, cytoplasm, and membrane. It is worth noting that an elastic material behavior with a Young modulus of $E=3 \mathrm{MPa}$ and a Poisson ratio of $v=0.3$ was considered for the network of microtubules [24]. The material parameters are listed in Table 1.

In addition, the Hugoniot and Tait equations of state were applied to the simulation in order to perfectly propagate the blast wave in the water cube.

\subsubsection{Hugoniot equation of state}

The Hugoniot equation of state was employed to describe the volumetric response of the cell under high-strain conditions. The shock response of many solid materials was well described by the Hugoniot relation, $U_{s}=C_{0}+s U_{p}$, between the shock wave velocity $U_{s}$ and the material velocity $U_{p} . C_{0}$ and $s$ are material parameters that could be obtained from the experiments. By combining this relation with the conservation of mass and momentum, the following equation of state could be extracted (Eq. (1)) [25]:

$$
P_{H}=\frac{\rho_{0} C_{0}^{2}\left(1-J_{H}\right)}{\left[1-s\left(1-J_{H}\right)\right]^{2}} .
$$

\subsubsection{Mie-Grüneisen equation of state}

The Mie-Grüneisen equation of state is quite relevant to the thermodynamic state. The thermodynamic state could rapidly change, and under the condition of adiabatic transition, the energy equation should not explicitly incorporate temperature. The governing equations could be solved if either the entropy or internal energy was specified as a function of pressure and density. It is the internal energy rather than temperature that is related to pressure and density (Eq. (2)) [26]:

$$
P=P_{H}\left(1-\frac{\Gamma_{0}}{2}\left(1-\frac{\rho_{0}}{\rho}\right)\right)+\Gamma_{0} \rho_{0} E,
$$

where $\Gamma_{0}$ is the Grüneisen parameter in the initial state.

\subsubsection{Generalized Maxwell model}

To complete the constitutive model description of the cell, a first-order generalized Maxwell model [27] was added to cell parts. The viscoelastic material parameters are given in Table 2 [2].
Table 2. Parameters for equation of state [5].

\begin{tabular}{lccccc}
\hline Material & $\begin{array}{c}\boldsymbol{\rho}_{\mathbf{0}} \\
\left(\mathbf{k g ~ m}^{-\mathbf{3}}\right)\end{array}$ & $\begin{array}{c}\boldsymbol{C}_{\mathbf{0}} \\
\left(\mathbf{m ~ s}^{-\mathbf{1}}\right)\end{array}$ & $\boldsymbol{s}$ & $\boldsymbol{\Gamma}_{\mathbf{0}}$ & $\begin{array}{c}\boldsymbol{\eta} \\
(\mathbf{P a . s})\end{array}$ \\
\hline Water & 1000 & 1450 & 1.99 & 0.1 & 0.001 \\
Cytoplasm & 1000 & 1450 & 1.99 & 0.1 & 1.08 \\
Nucleus & 1000 & 1450 & 1.99 & 0.1 & 431.11 \\
\hline
\end{tabular}

In addition, $\rho_{0}=1000\left(\mathrm{kgm}^{-3}\right)$ and $\eta=$ 0.001 (Pa.s) were chosen for membrane and water, respectively.

\subsection{Simulation process}

The simulation was performed for 150 nanoseconds. The equations of state were used for propagation of the blast wave in the water. FE method was applied to numerically solve the simulation. The analysis of the independence of the results from the computational grids was performed. For this purpose, the mesh density was increased gradually and the accuracy of the result was obtained. To be more specific, grid density of each model part varied from 1.5 to 2 times. For instance, the number of membrane elements increased by 4000 units approximately. After a specific grid density, a significant change in the obtained results was not observed. Actually, the average error between different mesh densities was in the range of 0.01 to $0.02 \%$ for different parts. The numbers of elements for the model with different arrays are given in Table 3 . The fluid consisted of 125000 cubic elements. A straightforward solver (MUMPS) was employed to solve the solid domain. In this method, an automatic preordering algorithm was applied and the pivot threshold factor was considered 0.1. The numbers of iterations for solving coupled fluid-solid equations were considered to be 10 and 15 for each step. The number of iterations for each step in the fluid domain was considered to be 200. Additionally, the number of residuals was chosen to be $4 \mathrm{E}-10$.

In this study, Comsol 5.2a software was employed. Given that some of the constants used in this research have been extracted from laboratory experiments $[2,24]$, the sensitivity of the results to change in the constants was investigated. Therefore, the parameters were considered variable. For example, by changing the elasticity of the microtubules in the

Table 3. Type and number of elements used in the simulation.

\begin{tabular}{lcc}
\hline \multicolumn{1}{c}{$\begin{array}{c}\text { Type of } \\
\text { array }\end{array}$} & $\begin{array}{c}\text { Domain } \\
\text { elements }\end{array}$ & $\begin{array}{c}\text { Boundary } \\
\text { elements }\end{array}$ \\
\hline Crossing & 147710 & 9357 \\
Stellate & 69252 & 3656 \\
Random orientation & 78833 & 4894 \\
\hline
\end{tabular}




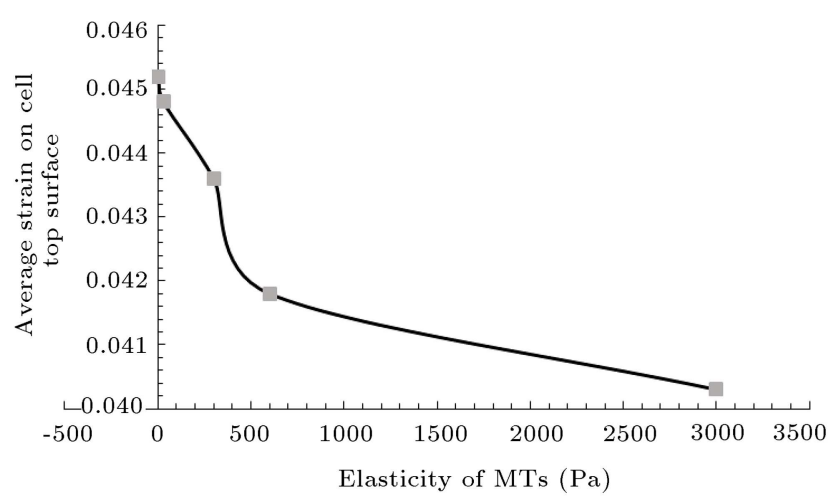

Figure 5. Average strain on cell top surface according to the elasticity of microtubules.

range of 1 to $30,000 \mathrm{MPa}$, the rate of average strain on the upper surface of the cell varied by less than $10 \%$ (Figure 5). As a consequence, the insignificant dependence of the results on the changes in the constants was clarified. It should be noted that the changes in the constants were made in the range of the reported values in the literature.

For model verification, the boundary conditions and the blast load were considered the same as [2]. In the research carried out by Jérusalem and Dao [2], the model was exposed to the constant pressure of $50 \mathrm{MPa}$ with a duration of 5 nanoseconds. The obtained results of the stress and pressure fields showed good agreement with Ref. [2], with an error less than $2 \%$. The cell response was captured without the presence of microtubules. The results for von Mises stress varied from 20 to $30 \mathrm{~Pa}$. This amount of von Mises stress showed that there was good agreement between the results of the two models. The von Mises stress in the cell is given in Figure 6.

\section{Results}

The results demonstrated that maximum pressure and maximum stress were reached at 73 and 65.5 nanoseconds (it is the time that the waves would reach the bottom of the cube). The effect of the presence of the

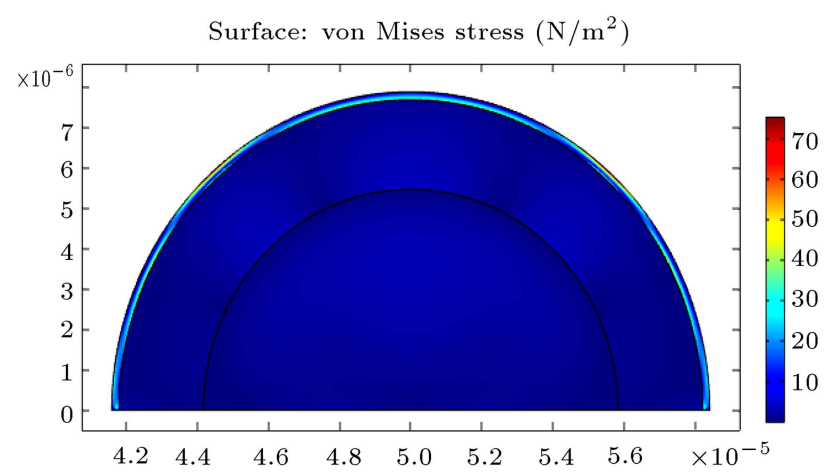

Figure 6. The von Mises stress in a cell without the network of microtubules.

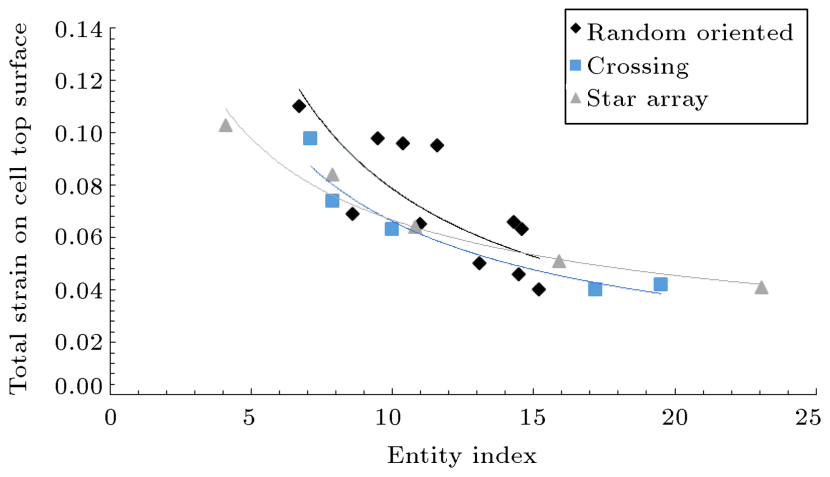

Figure 7. Total strain on top surface changes with an increase in entity index for different arrays.

microtubule network in three different arrangements inside the cytoplasm was investigated. By defining EI (Entity Index), as representative of the number of microtubules in each network [28], the effect of the presence of the microtubule network in the reduction of strain was investigated inside the cytoplasm. EI indicates the microtubule density inside the cell. By evaluating the results and comparing them with the strain values (Figure 7), it was conducted that the presence of the network of microtubules decreased the strain by 30 to $40 \%$. The strain that was experienced on the upper surface of the cell decreased, as shown in Figure 7. The strain was decreased from 0.12 in the control model to about 0.04 for the crossing network model, 0.046 for the stellate network, and 0.01 for the network with random orientation. The results for the density effect of the microtubules on the overall strain of the cell are depicted in Figure 7. Upon an increase in the density of the microtubules (the number of microtubules), the total cell strain was measured at the top level of the cell.

Besides, the von Mises stress distribution for microtubules is presented in Figures 8a to 8c. The von Mises stress within the membrane was decreased from $30 \mathrm{~Pa}$, in the cell without the presence of a microtubule network, to $15 \mathrm{~Pa}$, in the cell with the presence of the microbubbles, regardless of the array of the microtubule network. The von Mises stress inside the microtubules with crossing, stellate, and random orientation was 50,30 , and $35 \mathrm{~Pa}$, respectively.

\section{Discussion}

TBI has come to be known gradually as both longterm and short-term neurological deficits. Therefore, clarifying the molecular and cellular mechanisms of TBI is highly essential. Due to the lack of investigation on the sub-cellular mechanism of such damages to the brain, this research attempted to uncover the effects of blast load on the neuronal cell on the subcellular scale. In the research conducted by Jérusalem 
Surface: von Mises stress $\left(\mathrm{N} / \mathrm{m}^{2}\right)$

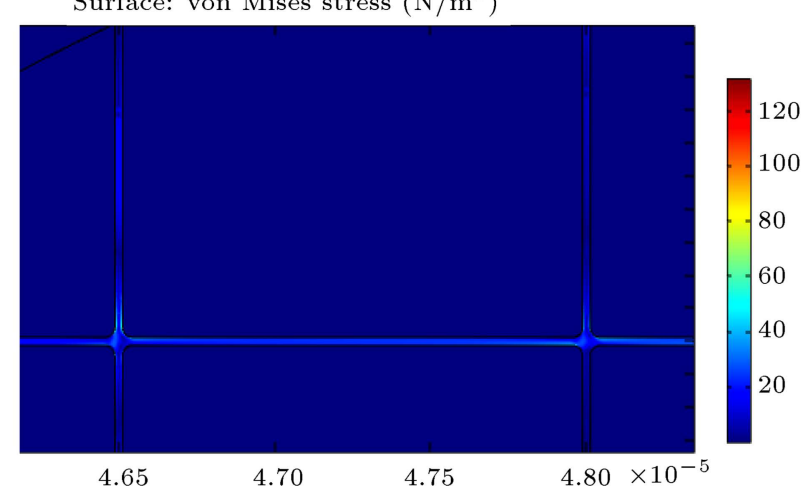

Surface: von Mises stress $\left(\mathrm{N} / \mathrm{m}^{2}\right)$

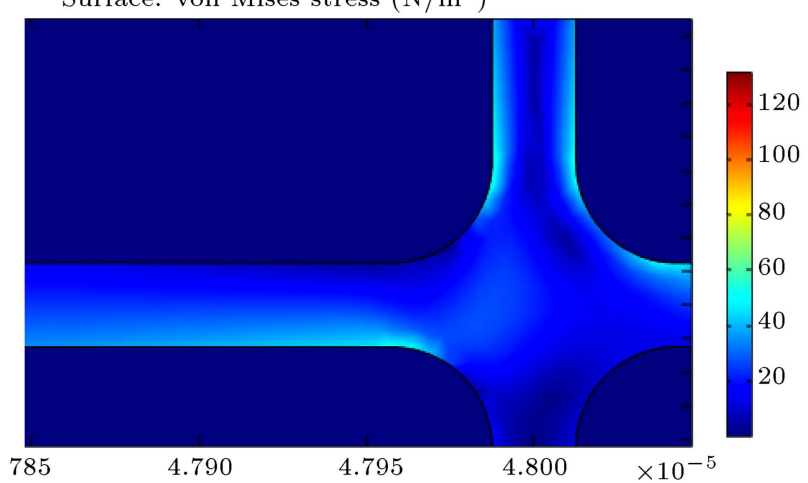

Figure 8a. The von Mises stress of the network of microtubules for crossing array $(\mathrm{Pa})$.

Surface: von Mises stress $\left(\mathrm{N} / \mathrm{m}^{2}\right)$

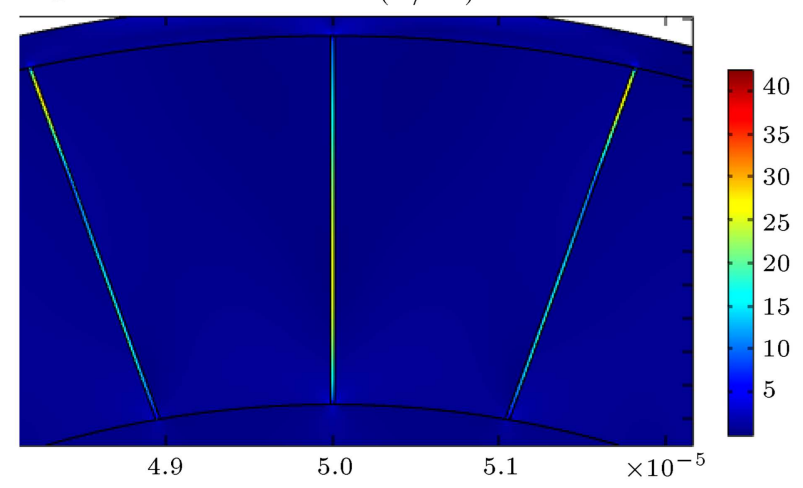

Surface: von Mises stress $\left(\mathrm{N} / \mathrm{m}^{2}\right)$

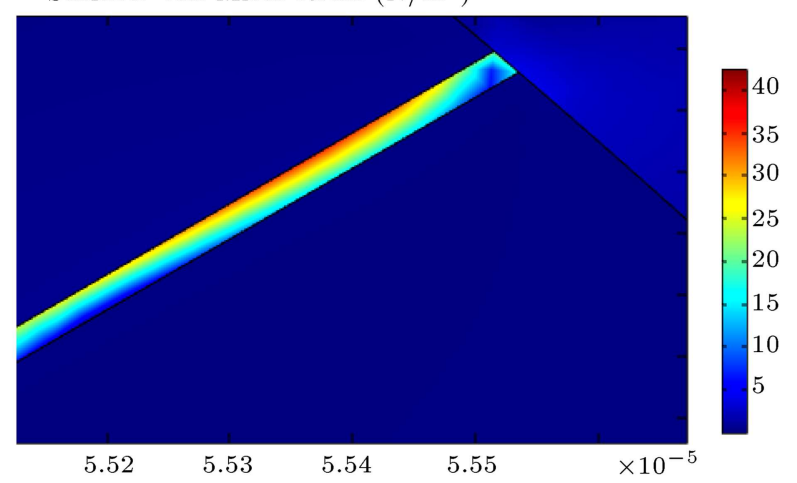

Figure $8 \mathrm{~b}$. The von Mises stress of the network of microtubules for stellate array $(\mathrm{Pa})$.
Surface: von Mises stress $\left(\mathrm{N} / \mathrm{m}^{2}\right)$

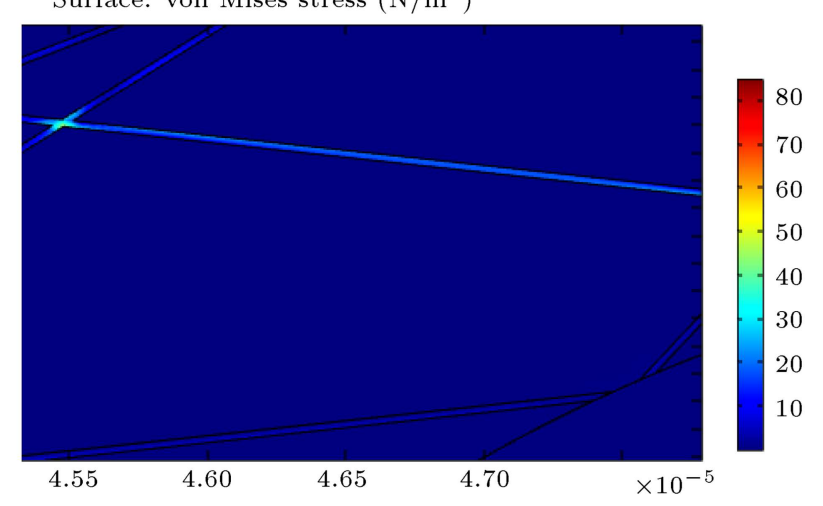

Surface: von Mises stress $\left(\mathrm{N} / \mathrm{m}^{2}\right)$

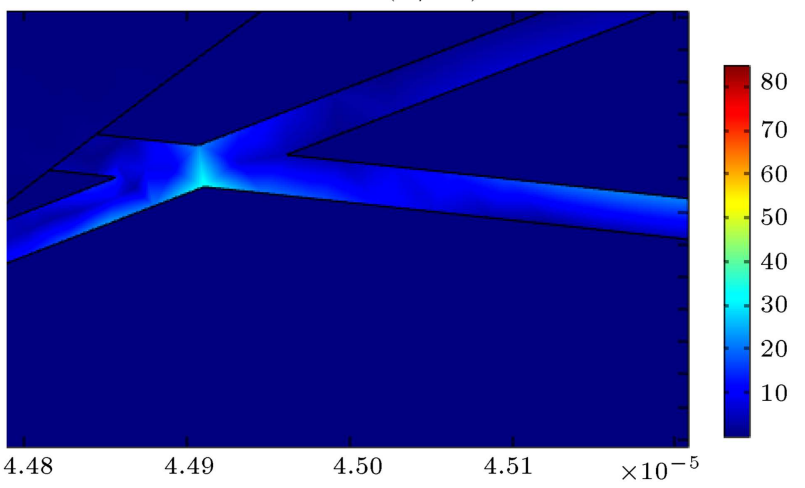

Figure 8c. The von Mises stress of the network of microtubules for a random orientation array $(\mathrm{Pa})$.

and Dao [2], the neuronal cell consisted of the three main parts as nucleus, membrane, and cytoplasm. In addition to the above-mentioned parts, the network of microtubules with different arrays, including crossing, stellate, and random orientation arrays, was considered in the present study.

Figure 7 indicates the role of the microtubule networks in reducing the strains experienced at the top level of the cell. As a result, sub-cellular components of the neural cell must be considered in the computational simulation research. Finally, the existence of the microtubules reduced both the strain of the upper surface of the cell and the least amount of stress experienced by different parts of the cell, especially cytoplasm and membrane. According to the results of the von Mises stress, much of the stress was carried by microtubules (regardless of their arrangements). Therefore, the critical role of the microtubules as a sub-cellular component of the cellular skeleton was determined. Furthermore, a $50 \%$ reduction in von Mises stress in the cell membrane emphasized this issue. The membrane in the blast load is the first part of the cell that would react. The change in permeability of the membrane and membrane disruption in large percentage might be the first response of the cell under blast load; in fact, the cascade of damage to the cell would be initiated with membrane damage. A transient increase 
in the permeability of neuronal plasma membrane has been reported by in-vitro studies, which became less intense after $10 \mathrm{~min}$. The loss of cell and synaptic disconnections has been contributed to the secondary cascades of damage. The response of a neuronal cell to blast load is highly complex. For instance, a network of neurons could be morphologically healthy, but electro physiologically dysfunctional which would lead to cell dysfunction or death [29]. Therefore, the importance of considering some components of the cellular skeleton of the cell was correctly addressed.

Figure 7 shows two points to be noticed: first, for a random orientation array, the overall strain decreased sharply due to an increase in the surface density of microtubules. Thus, this behavior was largely unreliable for the model. Biological evidence [30] also reflected the geometric mismatch of the random orientation array with the real network of microtubules. In fact, the microtubules were branched out around the nucleus and continued towards the membrane in real; second, in the crossing model, although there was still a problem of non-compliance with biological evidence [30], the strain was estimated to decrease with a slight slope. Ultimately, with an increase in the surface density of the microtubules, the strain of the cell converged to a certain range. Of course, there were some problems with crossing array including the fact that the values of the strain are almost out of a reasonable range and the diagram predicted the strain less than what actually could be. The last issue could be due to the very regular structure of this network. Finally, the diagram of cell strain in the presence of a stellate array was considered. This type of network arrangement has very high compatibility with the biological evidence [30], and the overall strain behavior for the cell in the presence of the stellate network was also within the acceptable range. Furthermore, its diagram had a logical slope (Figure 7) and the main reason for more rational results of the stellate array could be the fact that this network was more similar to the real network of microtubules inside the cytoplasm.

According to Figures $8 \mathrm{a}$ to $8 \mathrm{c}$, the numerical analysis clearly indicated that the presence of a network of microtubules would reduce the stress inside the cell. The decrease in cell stresses, regardless of variation in the arrangement of the network of microtubules, was obvious for all arrays (crossing, stellate, and random orientations) compared to the original cell model (control model). The results became more interesting when the internal effects of the microtubule networks were taken into account. As predicted earlier, due to the higher strength of the microtubule, much of the stress was carried by these sub-cellular parts. The reason behind this phenomenon is rooted in the fact that when composite material is under one-dimensional strain (such as a beam that is under pressure or strain from both ends and constituent materials are in parallel with the applied force), the member with a higher stiffness coefficient will tolerate greater stress, considering linear elasticity and the uniformity of the strain in the members.

Eventually, blast load was simulated similar to the laboratory research done by Sondén et al. [19] The results showed that this amount of pressure was sufficient to cause damage from grade 1 to 3 . This phenomenon is extremely dangerous to the integrity of the cell membrane. The values of pressure and stress could be sufficient for severe neural injury, even brain damage and fracture of the skull bone. The other serious consequences may include the damage to the receptor's organization, transfer of organs to the membrane, associated cellular skeleton components, and cell migration [2].

This research has been subjected to a number of constraints. First, wide ranges of the mechanical properties of the cell were reported in the literature. On the other hand, the complexity of the neuronal cell made it difficult to provide a comprehensive response of the neuronal cell to the blast load. Furthermore, considering the sub-cellular components of the model with different dimensions increased the computational costs. For future investigations, a more intricate network of sub-cellular components could be simulated as sub-cellular components such as cellular skeleton. Besides, investigation of the effect of the electrical with biomechanical responses of the neuronal cell under blast loading could be interesting for future studies. In addition, studying bending and buckling behavior of the microtubules under loading would be a challenging topic for future studies.

\section{Conclusions}

Traumatic brain injury is a consequence of brain damage to sudden shock. Understanding the mechanism and effects of such a damage to the brain is important for treating injuries. In this research, a model of a neural cell was presented. This model consisted of four independent parts: nucleus, cytoplasm, membrane, and different arrays of microbubbles. The nucleus, cytoplasm, and membrane followed viscoelastic mechanical behavior. The mechanical behavior of the microtubules was considered as linear elastic material. It is noteworthy that the results of this research could be used in various fields, including the designing of helmet, treatment of diseases such as kidney stones, treatment of sports injuries, prevention of traumatic astronauts, and ultimately prevention and treatment in all the fields related to traumatic brain injury.

The innovation of this study was selecting cell parts and setting appropriate material parameters for each cell part. Furthermore, the effects of the 
sub-cellular components for the neuronal cell were investigated. Sub-cellular components included an interconnected network of microtubules inside the cytoplasm of the neuronal cell. Besides, three main parts of the cell (nucleus, membrane, and cytoplasm) were taken into account. The effect of the presence of the network of microtubules on a cellular scale response was investigated. By observing the overall strain of the cell, the presence of the network of microtubules, regardless of the type of arrangement, reduced the overall cell stress and strain. In this research, three types of crossing, random orientation, and stellate arrangements were employed to simulate the microtubule network. Moreover, the surface index for the network of microtubules was defined, and the effect of increasing the number of microtubules, as a parameter, on the overall strain in the upper surface of the cell was assessed. It was conducted that the network of microtubules played a key role in the overall resistance of the cell to external loading. Besides, based on the comparison of the results, much of the von Mises stress was tolerated by a network of microtubules inside the cytoplasm. In addition, a $50 \%$ reduction in the membrane von Mises stress indicated the importance of the presence of a cellular skeleton in the simulation of the neuronal cell. As a result, the model successfully monitored some of the intracellular phenomena that could occur during neuronal damage.

\section{Nomenclature}

$\begin{array}{ll}E & \text { Young modulus (MPa) } \\ v & \text { Poisson ratio } \\ \mu_{1} & \text { Shear modulus }(\mathrm{Pa}) \\ \tau_{1} & \text { Relaxation time }(\mathrm{s}) \\ \eta & \text { Viscosity }(\mathrm{Pa} . \mathrm{s}) \\ U_{s} & \text { Shock wave velocity }(\mathrm{m} / \mathrm{s}) \\ U_{p} & \text { Material velocity }(\mathrm{m} / \mathrm{s}) \\ E & \text { Internal energy }\left(\mathrm{m}^{2} \cdot \mathrm{kg} / \mathrm{s}^{2}\right) \\ F S I & \text { Fluid-Structure Interaction } \\ C_{0} & \text { Material parameter }(\mathrm{m} / \mathrm{s}) \\ s & \text { Material parameter } \\ P_{H} & \text { Pressure behind the shock front }(\mathrm{Pa}) \\ \rho_{0} & \text { Reference density ahead of the shock } \\ & \left.\text { (kg.m }{ }^{-3}\right) \\ J_{H} & \text { Jacobian behind the shock front } \\ P & \text { Final pressure (Pa) } \\ \Gamma_{0} & \text { Grüneisen parameter } \\ T B I & \text { Traumatic Brain Injury } \\ E I & \text { Entity Index }\end{array}$

\section{References}

1. Bernick, K.B. "Cell biomechanics of the central nervous system", Thesis, Massachusetts Institute of Technology, USA (2011).

2. Jérusalem, A. and Dao, M. "Continuum modeling of a neuronal cell under blast loading", Acta Biomater., 8(9), pp. 3360-3371 (2012).

3. Edwards, D.S. and Clasper, J. "Blast injury mechanism", In Blast Injury Science and Engineering, pp. 87-104, Springer (2016).

4. Bernick, K.B., Prevost, T.P., Suresh, S., et al. "Biomechanics of single cortical neurons", Acta Biomater., 7(3), pp. 1210-1219 (2011).

5. Eslaminejad, A., Farid, M.H., Ziejewski, M., et al. "Brain tissue constitutive material models and the finite element analysis of blast-induced traumatic brain injury", Sci. Iran., 25, pp. 3141-3150 (2018).

6. Shams, S., Haddadpour, H., Tuzandejani, H., et al. "Impact crushing behavior of foam-filled paraboloid shells using numerical and experimental methods", Sci. Iran., Trans B, 24(4), pp. 1912-1921 (2017).

7. Ganpule, S., Alai, A., Plougonven, E., et al. "Mechanics of blast loading on the head models in the study of traumatic brain injury using experimental and computational approaches", Biomech. Model Mechan., 12(3), pp. 511-531 (2013).

8. Laksari, K., Assari, S., Seibold, B., et al. "Computational simulation of the mechanical response of brain tissue under blast loading", Biomech. Model Mechan., 14(3), pp. 459-472 (2015).

9. Laksari, K., Sadeghipour, K., and Darvish, K. "Mechanical response of brain tissue under blast loading", J. Mech. Behav. Biomed. Mater., 32, pp. 132-144 (2014).

10. Taylor, P.A., Ludwigsen, J.S., and Ford, C.C. "Investigation of blast-induced traumatic brain injury", Brain Inj., 28(7), pp. 879-895 (2014).

11. Teferra, K., Tan, X.G., Iliopoulos, A., et al. "Effect of human head morphological variability on the mechanical response of blast overpressure loading", Int. J. Numer. Meth. Bio., 34(9), p. e3109 (2018).

12. Ganpule, S., Daphalapurkar, N., Cetingul, M., et al. "Effect of bulk modulus on deformation of the brain under rotational accelerations", Shock Waves, 28(1), pp. 127-139 (2018).

13. Tan, X., Przekwas, A., and Gupta, R. "Computational modeling of blast wave interaction with a human body and assessment of traumatic brain injury", Shock Waves, 27(6), pp. 889-904 (2017).

14. Rodríguez-Millán, M., Tan, L., Tse, K., et al. "Effect of full helmet systems on human head responses under blast loading", Mater. Design, 117, pp. 58-71 (2017).

15. Finan, J.D. "Biomechanical simulation of traumatic brain injury in the rat", Clin. Biomech., 64, pp. 114121 (2019). 
16. Palombo, M., Alexander, D.C., and Zhang, H. "A generative model of realistic brain cells with application to numerical simulation of the diffusion-weighted MR signal", NeuroImage, 188, pp. 391-402 (2019).

17. Lu, Y.C., Daphalapurkar, N.P., Knutsen, A.K., et al. "A 3D computational head model under dynamic head rotation and head extension validated using live human brain data, including the falx and the tentorium", Ann. Biomed. Eng., 47(9), pp. 1923-1940 (2019).

18. Ahmadi-Nejad Joushani, H., Vahidi, B., and Sabour, M.H. "Investigating the effects of microtubules in the neuronal cell response to the blast load using fluidstructure interactions method", Journal of Solid and Fluid Mechanics, 9(3), pp. 13-24 (2019) (in Persian).

19. Sondén, A., Svensson, B., Roman, N., et al. "Laserinduced shock wave endothelial cell injury", Laser Surg. Med., 26(4), pp. 364-375 (2000).

20. Jean, R.P., Chen, C.S., and Spector, A.A. "Finiteelement analysis of the adhesion-cytoskeleton-nucleus mechanotransduction pathway during endothelial cell rounding: axisymmetric model", J. Biomech. Eng., 127(4), pp. 594-600 (2005).

21. Mofrad, M.R. and Kamm, R.D., Cytoskeletal Mechanics: Models and Measurements in Cell Mechanics, Cambridge University Press, UK (2006).

22. O'Connor, C.M., Adams, J.U., and Fairman, J., Essentials of Cell Biology, Cambridge, MA: NPG Education, 1 (2010).

23. Zander, N.E., Piehler, T., Boggs, M.E., et al. "In vitro studies of primary explosive blast loading on neurons", J. Neurosci. Res., 93(9), pp. 1353-1363 (2015).

24. Barreto, S., Clausen, C.H., Perrault, C.M., et al. "A multi-structural single cell model of force-induced interactions of cytoskeletal components", Biomaterials, 34(26), pp. 6119-6126 (2013).

25. Drumheller, D.S., Introduction to Wave Propagation in Nonlinear Fluids and Solids, Cambridge University Press (1998).

26. Meyers, M.A., Dynamic Behavior of Materials, John Wiley \& Sons (1994).

27. Kaliske, M. and Rothert, H. "Formulation and implementation of three-dimensional viscoelasticity at small and finite strains", Comput. Mech., 19(3), pp. 228-239 (1997).

28. Felgner, H., Frank, R., Biernat, J., et al. "Domains of neuronal microtubule-associated proteins and flexural rigidity of microtubules", J. Cell Biol., 138(5), pp. 1067-1075 (1997).

29. Prado, G.R., Ross, J.D., DeWeerth, S.P., et al. "Mechanical trauma induces immediate changes in neuronal network activity", J. Neural Eng., 2(4), p. 148 (2005).

30. Mathieu, P.S. and Loboa, E.G. "Cytoskeletal and focal adhesion influences on mesenchymal stem cell shape, mechanical properties, and differentiation down osteogenic, adipogenic, and chondrogenic pathways", Tissue Eng. Part B Rev., 18(6), pp. 436-444 (2012).

\section{Biographies}

Hossein Ahmadi Nejad Joushani received his BS degree in Mechanical Engineering from University of Kerman, Kerman, Iran in 2014 and his MS degree in Aerospace Engineering from University of Tehran, Tehran, Iran in 2017.

Bahman Vahidi received his BS degree in Mechanical Engineering from K.N. Toosi University of Technology, Tehran, Iran in 2004 and his MS degree in biomedical engineering from Amirkabir University of Technology, Tehran, in 2007. He also received his $\mathrm{PhD}$ from the same university in Biomechanics in 2011. He is currently an Associate Professor of Biomedical Engineering at University of Tehran, Iran. His research interests include cell mechanics, tissue engineering, biofluid mechanics, Fluid-Structure Interactions (FSI), and Computational Fluid Dynamics (CFD). Dr. Vahidi awards and honors include the honor of being the distinguished PhD student elected by the Ministry of Science, Research, and Technology of I.R. Iran in 2012. He was also elected to use Dr. Kazemi Ashtiani grant of Iran National Elites Foundation in 2013.

Mohammad-Hossein Sabour obtained his BS degree in Mechanical Engineering from University of Tehran, Tehran, Iran in 1989 and his MS degree in Mechanical Engineering from Tarbiat Modarres University in 1993. He also received his PhD in Aerospace Engineering from Concordia University in 2005. He is currently an Associate Professor at the Department of Aerospace Engineering at University of Tehran, Iran. 